\title{
Projeto de Antenas Compactas para um Sistema de Localização usando Sinais de TV Digital
}

\author{
Anderson L. A. Oliveira, Lisandro Lovisolo, Michel P. Tcheou
}

Resumo-Este trabalho apresenta o desenvolvimento de antenas operando na faixa da TV Digital Brasileira, entre $470 \mathrm{MHz}$ e $860 \mathrm{MHz}$, para integração de um sistema de localização usando sinais de TV Digital. Características de projeto relevantes são a diretividade e o tamanho físico da estrutura. São discutidos modelos distintos de conjuntos de dipolos - o Log-Periódico de condutores lineares cilíndricos, o Log-Periódico compacto em micro-fita - e o uso da geometria fractal de Koch visando a miniaturização da estrutura.

Palavras-Chave-Antenas, Localização, TV Digital, Fractais.

Abstract-This paper presents the development of antennas operating in Brazilian Digital TV, which ranges from $470 \mathrm{MHz}$ to $860 \mathrm{MHz}$, to integrate a localization system using Digital TV signals. The directivity and antenna size are important design aspects. Different models of dipole antenna arrays are discussed for comparison purposes - the Log-Periodic of cylindrical linear conductors and the compact Log-Periodic microstrip - using Koch's fractal geometry for miniaturizing the structure.

Keywords-Antennas, Localization, Digital TV, Fractals.

\section{INTRODUÇÃO}

Os serviços baseados em localização têm crescido em quantidade e penetração e são cada vez mais utilizados por aplicações e finalidades diversas [1], [2]. Uma alternativa para aumentar a disponibilidade desses serviços é usar sinais de TV Digital para fins de localização a partir de sinais de oportunidade [3]. Em geral, sistemas de radiodifusão de TV digital visam prover uma intensidade de campo elétrico mínima suficiente para a recepção na área de cobertura. Nas redes de frequência única contemporâneas, múltiplas antenas são usadas para prover uma cobertura conjunta satisfatória na frequência de operação [4]. Nesse cenário, para estimar a posição do receptor, uma estratégia possível seria empregar antenas capazes de prover alguma informação dependente da posição e orientação. Isso pode ser obtido por antenas com alguma diretividade para obter uma assinatura de radiofrequência angularmente distribuída.

Uma antena é um dispositivo que capta a energia eletromagnética radiada e a guia por uma linha de transmissão (e vice-versa) [5]. Uma antena largamente utilizada para recepção de sinais de TV é a estrutura formada por um conjunto logperiódico de dipolos: uma rede de elementos radiantes em uma configuração elétrica e geométrica [6]. Esse tipo de antena é capaz de operar em uma larga faixa espectral. Neste trabalho, busca-se reduzir o tamanho de antenas receptoras na faixa de TV digital preservando as características de ressonância,

A. L. A. Oliveira, L. Lovisolo e M. P. Tcheou, Faculdade de Engenharia, Programa de Pós-Graduação em Engenharia Eletrônica (PEL), Universidade do Estado do Rio de Janeiro (UERJ), Rio de Janeiro - RJ, e-mail: aandradeao@gmail.com, lovisolo@eng.uerj.br, mtcheou@eng.uerj.br. visando uma grande diretividade de forma a capturar o padrão circular da intensidade de campo elétrico recebido de uma SFN (Single Frequency Network). Usando uma antena giratória, a intensidade é capturada em diferentes radiais de uma volta de $360^{\circ}$ em torno do eixo da antena; o vetor com as intensidades de campo elétrico medidas em diferentes direções (que podem ou não ser provenientes de distintas antenas da SFN) possibilita estimar a posição [7].

Neste trabalho, avalia-se o comportamento do conjunto logperiódico de antena em micro-fita com aumento do fator de preenchimento de espaço através de curvas fractais de Koch [8], [9]. Com isso, é possível obter uma antena compacta operando na faixa de $540 \mathrm{MHz}$ a $645 \mathrm{MHz}$ com diretividade máxima de $10,73 \mathrm{~dB}$. As simulações foram realizadas nos softwares 4Nec2 e Ansys HFSS [10], [11].

\section{A. Organização}

Nas seções a seguir são discutidos os projetos e os resultados das antenas estudadas e simuladas. São apresentadas as principais características de cada uma delas, bem como os resultados gerados através das simulações. Na Seção II, é apresentada a antena log-periódica composta por elementos cilíndricos. É adicionado um refletor de canto e observado o seu efeito sob a estrutura log-periódica. A Seção III apresenta uma estrutura log-periódica impressa em micro-fita. Essa tecnologia permite a confecção de estruturas menores, com maior facilidade de construção e custo reduzido. Na Seção IV, aplica-se a geometria fractal de Koch em uma antena linear impressa, de modo a reduções as dimensões físicas da antena. A Seção V apresenta os resultados obtidos. Por fim, a Seção VI traz as conclusões do trabalho.

\section{Antena Log-PeriódicA}

A estrutura log-periódica de dipolos é uma rede de elementos dipolos. Consideramos uma estrutura composta por dipolos convencionais (cilíndricos), que apresenta característica endfire (a irradiação é máxima na direção longitudinal). A estrutura geométrica é projetada para que a impedância de entrada e os diagramas de radiação variem periodicamente em função do logaritmo da frequência [5]. O comprimento de cada elemento e seu adjacente estão relacionados pelo fator $\tau$, chamado Razão Geométrica ou Fator de Escala [5],

$$
\tau=l_{n} / l_{n+1},
$$

onde $l_{n}$ é o comprimento do $n$-ésimo dipolo. Já os espaçamentos entre os elementos dipolo são dependentes do fator $\sigma$, chamado de Fator de Espaçamento [5],

$$
\sigma=a_{n} / 2 l_{n+1}
$$


em que $a_{n}$ é o espaçamento físico entre $n$-ésimo e $(n+1)$ ésimo elementos do arranjo.

Escolheu-se a antena log-periódica como ponto de partida para este estudo por apresentar uma resposta banda-larga e um padrão de radiação diretivo. Os fatores de escala e espaçamento são definidos em geral usando um método gráfico, a partir de curvas de diretividade que a mapeiam os valores de $\sigma$ e $\tau$ [5]. De posse das curvas de contorno da constante de diretividade versus $\sigma$ e $\tau$ para um conjunto log-periódico de dipolos, obteve-se $\sigma=0,17$ e $\tau=0,92$.

Definidos os valores dos parâmetros $\sigma$ e $\tau$ e conhecida a faixa alvo de frequências, é possível calcular o número de dipolos necessários e outros aspectos da estrutura como os comprimentos dos dipolos e o espaçamento entre eles. Calculase a largura de banda da estrutura via

$$
B_{s}=\frac{f_{\max }}{f_{\min }}\left[1,1+7,7 \frac{(1-\tau)^{2}}{\tan (\alpha)}\right],
$$

onde $f_{\max }$ é a frequência máxima de operação e $f_{\min }$ a mínima. Acima, o parâmetro $\alpha$ é o ângulo de abertura

$$
\alpha=\frac{180}{\pi} \tan ^{-1}\left(\frac{1-\tau}{4 \sigma}\right) .
$$

O comprimento axial total da estrutura é dado por

$$
L=\frac{\lambda_{\max }}{4}\left(1-\frac{1}{B_{s}}\right) \operatorname{cotg}(\alpha),
$$

onde $\lambda_{\max }$ é o comprimento de onda máximo.

Calcula-se a largura de banda, considerando $f_{\min }=$ $515 \mathrm{MHz}, f_{\max }=636 \mathrm{MHz}, \tau=0,92$ e $\alpha=6,7^{\circ}, \log$,

$$
B_{s}=\frac{636}{515}\left[1,1+7,7 \frac{(1-0,92)^{2}}{\tan \left(6,7^{\circ}\right)}\right] \cong 1,876 .
$$

Uma vez definida a largura de banda, obtém-se o número de elementos radiantes $N$ via [6]

$$
N=1-\frac{\ln \left(B_{s}\right)}{\ln (\tau)}=1-\frac{\ln (1,876)}{\ln (0,92)} \cong 8 .
$$

Os elementos radiantes de menor e maior comprimentos têm $13,157 \mathrm{~cm}$ e $21,698 \mathrm{~cm}$, respectivamente. O espaçamento médio entre os dipolos é de $5,681 \mathrm{~cm}$. A geometria foi construída no software 4Nec2 [12]. Em seguida, projetou-se um refletor do tipo canto com abertura de $60^{\circ}$, com o intuito de aumentar a diretividade. A estrutura simulada pode ser vista na Fig. 1 e os diagramas de radiação, nas frequências de 563 MHz e $623 \mathrm{MHz}$, na Fig. 2.

Observando os diagramas de radiação da antena logperiódica de elementos radiantes cilíndricos com refletor, Fig. 2, percebe-se, nas frequências de $563 \mathrm{MHz}$ e $623 \mathrm{MHz}$, que tal estrutura apresenta diretividade com larguras de feixes em torno de $45^{\circ}$ com ganho próximo a $12 \mathrm{~dB}$.

\section{ANTENA LOG-PERIÓDICA LINEAR IMPRESSA}

O uso de antenas de micro-fita em diversas aplicações é potencializado pela facilidade de construção, a leveza e o reduzido custo de fabricação das mesmas [13]. Entretanto, geralmente, tais antenas apresentam banda estreita. A fim de

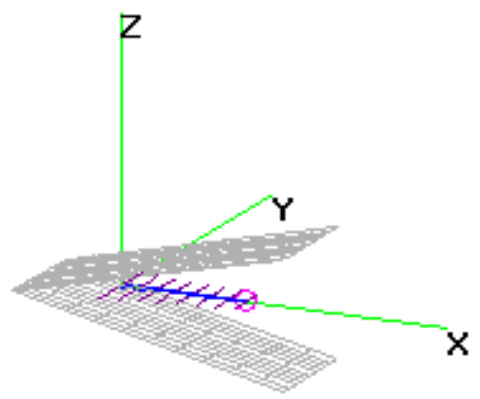

Fig. 1. Antena log-periódica horizontal simulada no software $4 \mathrm{Nec} 2 \mathrm{com}$ refletor de canto horizontal com abertura de $60^{\circ}$.
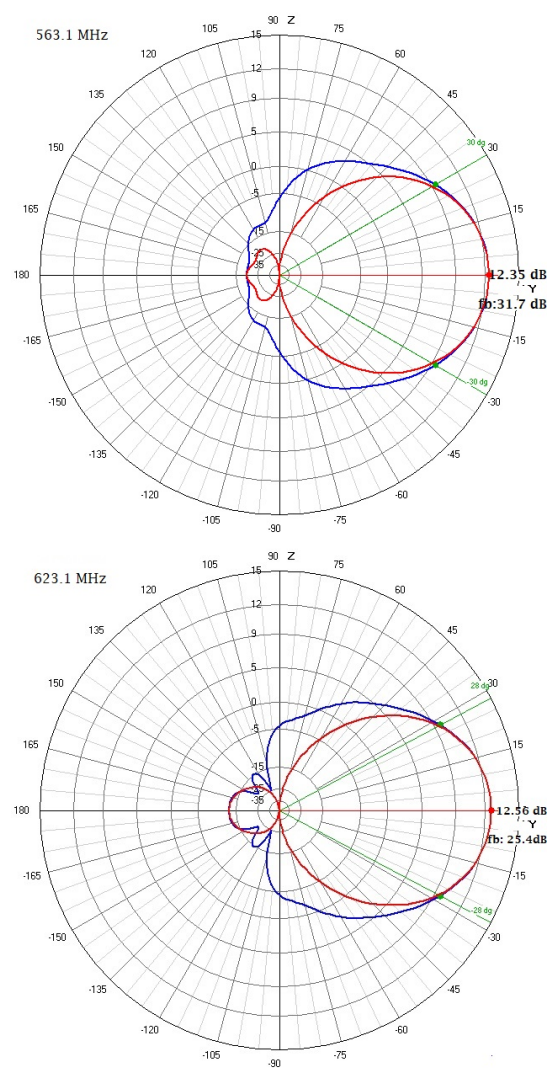

Fig. 2. Diagramas de Radiação da Log-Periódica de dipolos cilíndricos nos planos azimutal e de elevação nas frequências $563 \mathrm{MHz}$ (acima) e $623 \mathrm{MHz}$ (abaixo).

contornar essa situação, pode-se empregar a topologia de antenas log-periódicas lineares em circuito impresso compostas por um substrato, em geral de material dielétrico, coberto por lâminas de metal em sua superfície.

As antenas de micro-fita podem ser alimentadas por uma variedade de métodos, classificados como conectados e não conectados. Neste trabalho, consideramos a antena conectada: a fonte de RF é ligada ao patch (parte radiante) usando uma linha de micro-fita; a linha de micro-fita é composta por um condutor, geralmente muito menor que a largura do patch. A antena apresenta duas fitas paralelas impressas em faces opostas do substrato. Ela é de fácil fabricação, porém à medida que a espessura do substrato aumenta há um incremento das 
ondas de superfície e da radiação espúria da alimentação.

A largura da linha de alimentação deve ser tal que

$$
Z_{\text {ofp }}=2 Z_{\text {omicrofita }}
$$

onde $Z_{\text {ofp }}$ é a impedância característica da linha de fitas paralelas, e

$$
Z_{\text {omicrofita }}=\frac{120}{2 \sqrt{\epsilon_{r e}}} \ln \left(\frac{F_{1}}{u}+\sqrt{1+\frac{4}{u^{2}}}\right),
$$

é a impedância característica de uma micro-fita impressa em substrato com metade da espessura $h$ do laminado utilizado na confecção da linha. Acima,

$$
\begin{aligned}
& F_{1}=6+(2 \pi-6) e^{-\left(\frac{30,66}{u}\right)^{0,7528},} \\
& \epsilon_{r e}=\frac{\epsilon_{r}+1}{2}+\frac{\epsilon_{r}-1}{2}\left(1-\frac{10}{u}\right)^{-a b},
\end{aligned}
$$

onde $\epsilon_{r e}$ é a constante dielétrica efetiva da micro-fita, $\epsilon_{r}$ é a constante dielétrica do substrato. Os valores de $u, a$ e $b$ são dados por

$$
a=1+\frac{1}{49} \ln \left(\frac{u^{4}+\left(\frac{u}{52}\right)^{2}}{u^{4}+0,432}\right)+\frac{1}{18,7} \ln \left(1+\left(\frac{u}{18,1}\right)^{3}\right)
$$$$
b=0,564\left(\frac{\epsilon_{r}-0,9}{\epsilon_{r}-0,3}\right)^{0,053},
$$

$u=\frac{2 W}{h}$,

sendo $W$ a largura da linha e $h$ a sua espessura [13].

Para o projeto de uma antena log-periódica em circuito impresso, inicialmente, obtém-se a largura da fita de alimentação da antena a partir das equações (8) - (14), obtendo uma largura de 4,5 mm para a linha de alimentação da micro-fita. Usando as curvas de contorno da diretividade versus $\sigma$ e $\tau$, obtêm-se $\sigma=0,157$ e $\tau=0,865$. Definidos esses parâmetros, obtémse o ângulo de abertura $\alpha=12,13^{\circ}$. Na simulação, usou-se um substrato dielétrico Taconic TLC-338 de permissividade elétrica $\epsilon_{r}=3,3$ e espessura $h=1,524 \mathrm{~mm}$ [8].

\section{A. Miniaturização da Log-Periódica Impressa}

Usando como referência a antena log-periódica impressa, a fim de se reduzir o seu comprimento, projetou-se e simulou-se uma log-periódica com 3 elementos radiantes, diminuindo a banda ressonante original, de frequências mínima e máxima iguais a $515 \mathrm{MHz}$ e $680 \mathrm{MHz}$, para a faixa entre 540 $\mathrm{MHz}$ e $645 \mathrm{MHz}$. A configuração final é apresentada na Fig. 3 e os diagramas de radiação estão na Fig. 4. Nessa configuração, percebe-se perda na diretividade da antena. $\mathrm{O}$ padrão de radiação tem como largura de feixe um ângulo de aproximadamente $65^{\circ}$ e ganho de $10 \mathrm{~dB}$.

\section{ANTENA FRACTAL}

\section{A. A Curva de Koch}

Mandelbrot cunhou o termo fractal para referir-se a objetos geométricos infinitamente subdivisíveis auto-similares, onde cada subdivisão se assemelha ao todo [14]. A curva de

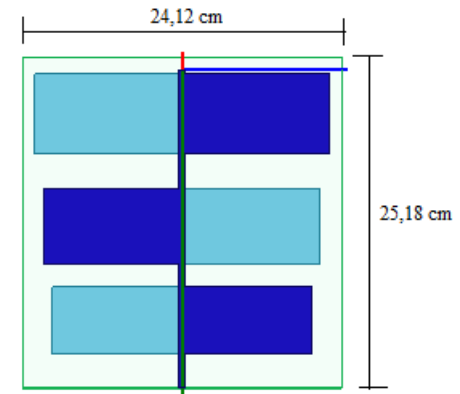

Fig. 3. Antena log-periódica com três elementos ressonantes na faixa de frequências entre $540 \mathrm{MHz}$ e $645 \mathrm{MHz}$.
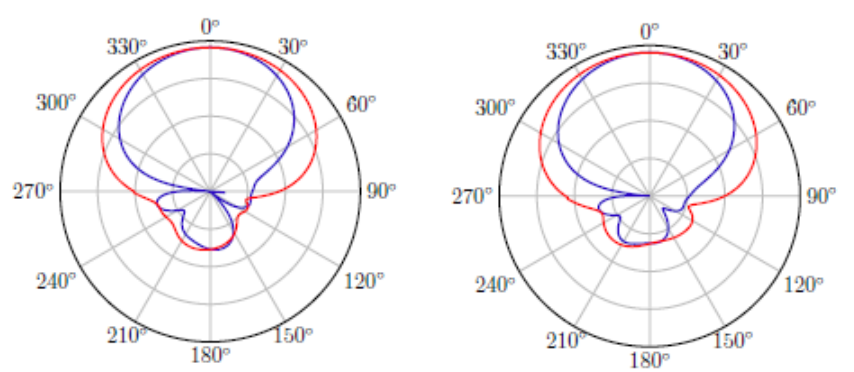

Fig. 4. Diagramas de Radiação da antena Log-Periódica impressa em microfita nos planos azimutal e de elevação nas frequências $563 \mathrm{MHz}$ e $623 \mathrm{MHz}$.

Koch, apresentada por Niels Fabian Von Koch, é uma curva geométrica sem tangentes e um dos primeiros fractais a serem descritos [14]. Para construí-la [9]:

1) Inicia-se a partir de uma reta qualquer de comprimento unitário;

2) Cada segmento é dividido em três segmentos menores;

3) O segmento central é substituído por um triângulo equilátero sem a base, transformando o segmento original numa curva poligonal com quatro segmentos de comprimentos iguais;

4) Aplicam-se os passos 2 e 3 a cada segmento da curva poligonal resultante por $n$ iterações.

Cada segmento da primeira iteração, $K_{1}$, tem um terço do comprimento linear, $l$ de $K_{0}$, assim passam a existir quatro retas com comprimentos de $\frac{l}{3}$ totalizando um comprimento que vale $\frac{4}{3} l$. A cada iteração, o mesmo procedimento é aplicado em todos os segmentos de reta. A Fig. 5 ilustra o processo. O comprimento físico total da curva de Koch, cresce $\frac{4}{3}$ a cada iteração.

\section{B. Projeto da Log-Periódica Fractal de Koch de Segunda Iteração}

Os mesmos procedimentos de cálculo de parâmetros construtivos dos dipolos da antena log-periódica linear são usados para a antena log-periódica fractal [8]. Entretanto, para a estrutura fractal são usadas as regras de formação das curvas de Koch [9], permitindo uma redução do comprimento físico dos elementos individuais. Aplicamos o contorno de Koch de segunda iteração, uma vez que já implica em uma considerável redução do comprimento físico dos elementos individuais e, 


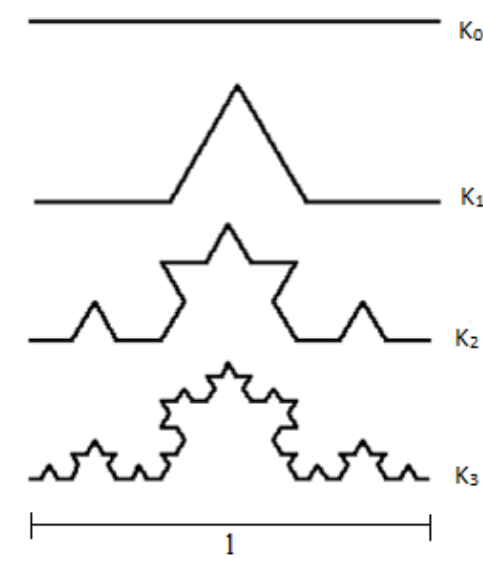

Fig. 5. As três primeiras iterações das curvas de Koch de mesmo comprimento linear $l$.

a cada iteração, aumenta-se a complexidade de construção da estrutura. Com essa estratégia, é possível reduzir em aproximadamente $60 \%$ a área total da antena log-periódica linear impressa como se observa ao comparar as dimensões indicadas na Fig. 6 e na Fig. 3. A antena (Fig. 6) apresenta padrão endfire de irradiação. Isso é possível por ela não possuir plano de terra e, dessa forma, a estratégia utilizada para a defasagem das correntes entre cada elemento radiante consiste em imprimir a metade de cada dipolo em faces opostas do substrato [13].

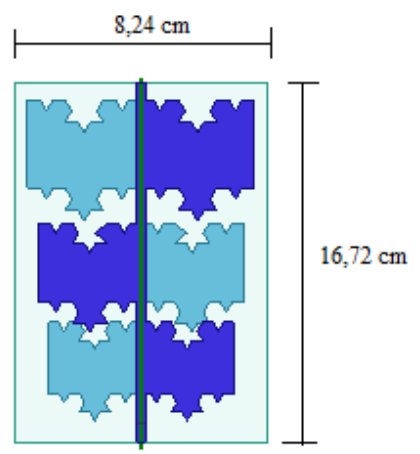

Fig. 6. Antena log-periódica impressa usando contornos fractais da curva de Koch.

\section{Resultados}

Visa-se reduzir da área física da estrutura e deve-se verificar se a antena projetada opera na banda de interesse. Para isso, analisa-se a curva de Perda de Retorno. A Perda de Retorno é uma medida da potência que é devolvida à fonte por uma carga reflexiva, isto é,

$$
R L=10 \log _{10} \frac{1}{\left|\Gamma_{L}\right|^{2}},
$$

onde $\Gamma_{L}$ é o coeficiente de reflexão da carga considerada [6]. Admite-se que a ressonância e a consequente transmissão da onda eletromagnética através da superfície da antena ocorre a perda de retorno inferiores a $-10 \mathrm{~dB}$.
O diagrama de radiação tridimensional e a curva de Perda de Retorno para a frequência de $563 \mathrm{MHz}$ são apresentados na Fig. 7. Já os diagramas planares de radiação em $563 \mathrm{MHz}$ e $623 \mathrm{MHz}$ podem ser vistos na Fig. 8 .
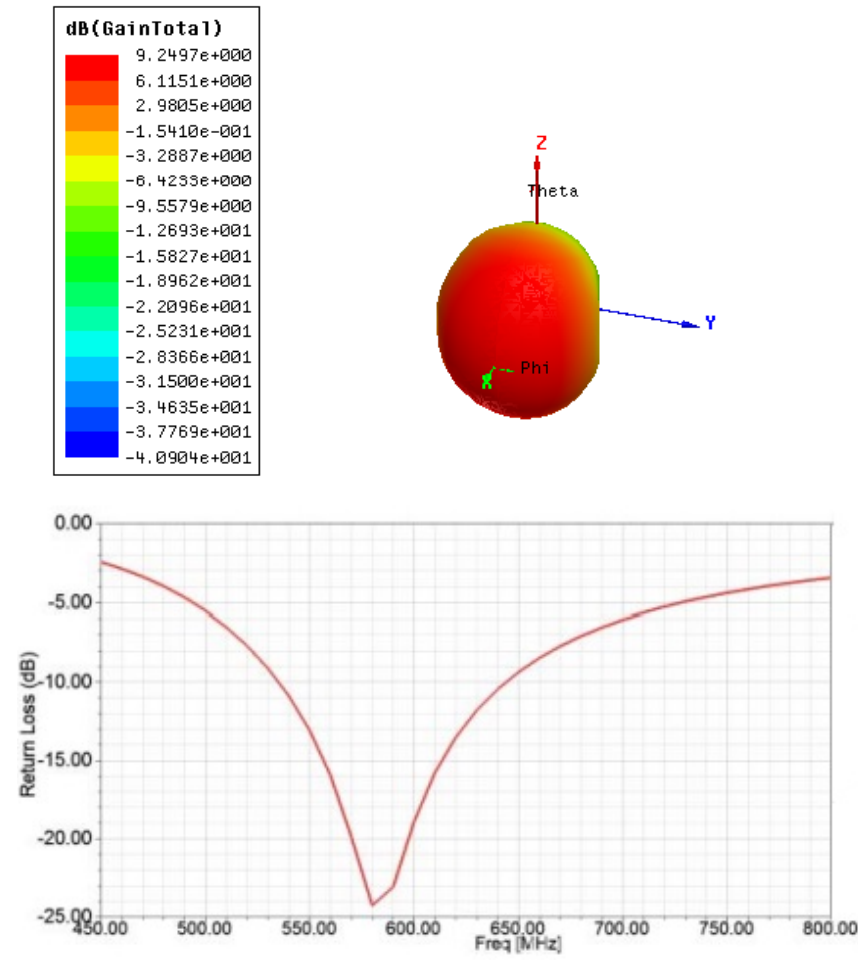

Fig. 7. Diagrama de radiação tridimensional (acima) e perda de retorno (baixo) da antena Log-Periódica de Koch para a frequência de $563 \mathrm{MHz}$.
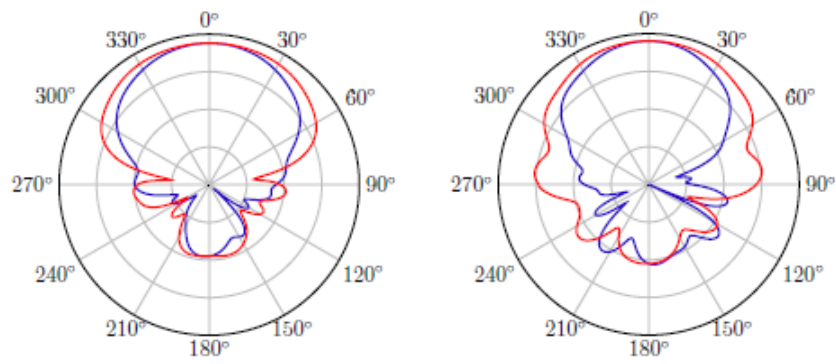

Fig. 8. Diagramas de radiação da antena Log-Periódica de Koch nos planos azimutal e de elevação nas frequências $563 \mathrm{MHz}$ e $623 \mathrm{MHz}$.

A curva de Perda de Retorno (Fig. 7) da antena logperiódica de Koch apresenta resposta satisfatória (igual ou inferior a $-10 \mathrm{~dB}$ ) na faixa de interesse do projeto. Nos diagramas de radiação (Fig. 8), verifica-se que, embora haja uma maior radiação nas costas da antena do que a observada para a antena log-periódica linear de micro-fita, a razão frentecostas é maior que $15 \mathrm{~dB}$. Os ganhos da antena em ambas as frequências analisadas são de aproximadamente $10 \mathrm{~dB}$ e as larguras de feixe estão entre $70^{\circ}$ e $80^{\circ}$.

A estrutura final do projeto, usando o conjunto log-periódico de Koch, resulta em uma antena com dimensões físicas de 8,24 $\mathrm{cm} \times 16,72 \mathrm{~cm}$, operando em uma banda de 540 a $645 \mathrm{MHz}$, 
com um ganho de $9,25 \mathrm{~dB}$, diretividade de $10,73 \mathrm{~dB}$, e perda de retorno $\left(S_{11}\right)$, em $563 \mathrm{MHz}$, igual a $-24 \mathrm{~dB}$. Ao reduzir o comprimento total de cada dipolo que compõe a rede fractal, espera-se uma redução do ganho da antena. No entanto, o coeficiente de reflexão obtido encontra-se abaixo de $-10 \mathrm{~dB}$.

Em [9], as antenas log-periódicas fractais projetadas de primeira e segunda iteração apresentaram coeficientes de reflexão superiores a $-10 \mathrm{~dB}$ na faixa de projeto, não sendo propostas soluções que contornem esta limitação. Já em [8] os contornos fractais aplicados nos dois níveis diferentes foram do tipo Koch quadrado. Essa estratégia permitiu manter a antena compacta o suficiente na banda de interesse (entre 1 $\mathrm{GHz}-10 \mathrm{GHz}$ ).

Neste trabalho, aplicaram-se os contornos fractais do tipo Koch retangular, tendo como referência uma antena logperiódica impressa retangular, preterindo a Koch quadrada. Dessa maneira, pode-se operar na faixa de frequências da TV Digital, o que viabiliza a utilização da antena para o sistema de localização alvo. O coeficiente de reflexão da antena proposta apresentou valor inferior a $-10 \mathrm{~dB}$ na faixa de operação.

\section{CONCLUSÕES}

Neste trabalho, aplicaram-se os contornos fractais do tipo Koch retangular no projeto de uma antena log-periódica impressa retangular na faixa de frequências da TV Digital com diretividade para utilização em sistema de localização a partir de sinais de TV. O uso da antena fractal de Koch não acarretou prejuízos de ganho ou diretividade, relativamente à antena original, permitindo, conforme pretendido, obter uma estrutura fisicamente menor. Verificou-se que a estrutura fractal projetada e simulada responde conforme desejado. Obtémse assim uma antena de dimensão relativamente pequena operando na faixa de UHF com diretividade suficiente para emprego no sistema de localização. Futuramente, pretende-se imprimir a estrutura simulada e realizar testes experimentais. Deseja-se ainda avaliar o comportamento da antena ao se aplicar curvas de Koch de maiores níveis e outras geometrias fractais.

\section{Agradecimentos}

O presente trabalho foi realizado com apoio da Coordenação de Aperfeiçoamento de Pessoal de Nível Superior - Brasil (CAPES) - Código de Financiamento 001, do Conselho Nacional de Desenvolvimento Científico e Tecnológico (CNPq) e da Fundação de Amparo à Pesquisa do Estado do Rio de Janeiro (FAPERJ).

\section{REFERÊNCIAS}

[1] Rafael Saraiva Campos and Lisandro Lovisolo. RF positioning: fundamentals, applications, and tools. Artech House, 2015.

[2] Seyed Reza Zekavat, R Michael Buehrer, Gregory D Durgin, Lisandro Lovisolo, Zhonghai Wang, Shu Ting Goh, and Ahmad Ghasemi. An overview on position location: Past, present, future. International Journal of Wireless Information Networks, pages 1-32, 2021.

[3] Jie Huang. Positioning techniques with signal of opportunity: a focus on digital television systems. 2014.

[4] Christian FF Rodrigues and Lisandro Lovisolo. Heuristic-based location allocation of single frequency network stations. IEEE Transactions on Broadcasting, 2020.
[5] Constantine A Balanis. Antenna theory: analysis and design. John wiley \& sons, 2005

[6] L. C. Esteves. Antenas-Teoria Básica e Aplicações. McGraw-Hill, 1rd edition, 1987.

[7] Tayssa Dominguez, Lisandro Lovisolo, Michel Pompeu Tcheou, an Rafael Saraiva Campos. Localização de dispositivos usando correlação de assinaturas de radiofrequência em redes isdb-t de frequência Única. 2019.

[8] V.A. de A. Filho. Arranjos log-periódicos em microfita com elementos fractais de Koch. UFRN; Natal-RN, 2010.

[9] V. S. Wildner and M. G. Vanti. Projeto de antena log-periódica de Koch fractal com três iterações de Kock para TV digital. SBrT, 2013.

[10] Z. Cendes. The Development of HFSS. USNC-URSI Radio Science Meeting.Fajardo, 2016.

[11] A. C. Silva, Nobre B. L., C. C. R Albuquerque, and C. P. Silva. Design de antenas planares utilizando o high frequency structure simulator (HFSS) da ANSYS®. XXXVI SBrT, 2018.

[12] R.M. Martins. Análise de softwares de modelagem de antenas para cursos técnicos e de engenharia de telecomunicações. $5^{\circ}$ Simpósio de Integração Científica e Tecnológica do Sul Catarinense.

[13] M Pozar, D. Microwave Engineering, volume 1. John Wiley \& Sons Ltd, 4rd edition, 2005.

[14] B.B. Mandelbrot. The Fractal Geometry of Nature. W. H. Freeman and Company, 1982. 